\title{
Molecular identification of cholesterol-lowering lactic acid bacteria isolated from cocowater kefir
}

\author{
Ruel Valerio de Grano ${ }^{1}$, Emma Teresa Carmela Aportadera ${ }^{1}$, Mark Kevin Devanadera ${ }^{1,3}$, \\ Myla R. Santiago ${ }^{1,2}$, E Leslie Michelle Dalmacio ${ }^{4 *}$ \\ ${ }^{1}$ Department of Biochemistry, Faculty of Pharmacy, ${ }^{2}$ Research Center for the Natural and Applied \\ Sciences, $\mathcal{E}{ }^{3}$ The Graduate School, University of Santo Tomas, 1015 Manila, Philippines; \\ ${ }^{4}$ Department of Biochemistry and Molecular Biology, \\ University of the Philippines-Manila, 1000 Manila, Philippines
}

\begin{abstract}
High cholesterol levels are explicitly linked to cardiovascular diseases which are the leading causes of death worldwide. Treatments such as statins often have undesirable side effects or high costs. Probiotics have been gaining popularity as a possible alternative for management of various illnesses. Cocowater kefir has exhibited cholesterol-lowering ability, attributed to the lactic acid bacteria present. Thus, the study aims to identify the specific strain of lactic acid bacteria involved to ascertain the cholesterol-lowering property and probiotic potential of microorganisms isolated from cocowater kefir. Lactic acid bacteria were isolated and purified from cocowater kefir and screened for its cholesterol lowering activity through colorimetric cholesterol assay (CHOD-PAP). The cocowater kefir strain of lactic acid bacteria showed significant cholesterol-lowering property with respect to a standard microorganism. The cholesterol concentration was $66.27 \mathrm{mg} / \mathrm{dL}$ and $29.00 \mathrm{mg} / \mathrm{dL}$ after 24 and $48 \mathrm{~h}$ incubation with isolated lactic acid bacteria. The cholesterol inoculated with lactic acid bacteria was computed and a decrease in cholesterol levels to 8.31 and $43.06 \mathrm{mg} / \mathrm{dL}$ at 24 and $48 \mathrm{~h}$ incubation was obtained. The genomic DNA of lactic acid bacteria isolated was extracted and sequenced. The strain was identified through sequence analysis as Lactobacillus plantarum with $99 \%$ identity. The DNA sequence of the isolated lactic acid bacteria was deposited in the GenBank with a definition of L. plantarum strain KFR1, $16 \mathrm{~S}$ ribosomal RNA gene, partial sequence with an accession number of KT581997.
\end{abstract}

Keywords: probiotics, cholesterol, cocowater kefir, L. plantarum

\section{INTRODUCTION}

Elevated serum cholesterol levels have been widely recognized as a contributory risk factor in the development of cardiovascular diseases, such as coronary heart disease and

*To whom correspondence should be addressed lesmdmc@gmail.com atherosclerosis [1]. Consumption of certain cultured dairy products has been shown to result in cholesterol reduction. Studies have indicated that metabolites produced by starter cultures such as lactic acid bacteria (LAB) inhibit cholesterol synthesis in vitro [1-7] and in vivo in mice, pigs, and humans [7]. This may be due to bile salt hydrolase [3], the incorporation of cholesterol into bacterial cell membrane 
during growth [4], the inhibition of cholesterol synthesis by metabolites produced by starter culture bacteria [2], the disruption of the cholesterol micelle and/or the binding of cholesterol to cellular surface [5].

Kefir, an alcoholic milk beverage produced by fermentation of kefir grains, possesses antitumor, antimutagenic, antioxidant, and hypocholesterolemic effects [7]. Despite the microbial diversity found in kefir samples, common LAB strains predominate such as Lactobacillus acidophilus, Lactobacillus plantarum, Lactobacillus kefiranofaciens had been isolated from kefir grains [8]. Kefir grains are composed of peptide and sugar prebiotics such as lactacin, bacteriocins, kefiran which facilitate a stable microbial ecology of bacterial and yeast species working in symbiosis. These grains were supposedly developed in milk stored in animal skin, intestine or bladder containers [8]. Coconut water was used as a substitute substrate instead of milk. Its sugar component serves as substrate for microbial growth including amino acids, aromatic compounds and vitamins [9]. To date, this is the first study to isolate and identify cholesterol lowering LAB from kefir using Philippine coconut water as substrate. This study aims to isolate, characterize, and identify the probiotics that would exhibit cholesterollowering properties and compare it with the standard strain that exhibit cholesterollowering such as L. plantarum BS25

\section{EXPERIMENTAL}

Fermentation. Coconut water was used as a substitute substrate instead of milk. Kefir grains were inoculated in an Erlenmeyer flask containing coconut water with a ratio of $0.5 \mathrm{~g}$ kefir grains in $10 \mathrm{~mL}$ coconut water and incubated for $72 \mathrm{~h}$ at $25^{\circ} \mathrm{C}$ and the media was changed every $24 \mathrm{~h}$. The biomass was harvested by filtering the kefir grains from media with sterile gauze, air-dried, and weighed.
Isolation of lactic acid bacteria. A loopful of cocowater kefir was inoculated on the surface of MRS agar plate and incubated at room temperature for $24 \mathrm{~h}$. After incubation, three individual colonies with the same morphology were chosen randomly and picked. The colonies were separately sub-cultured on sterile MRS broth and on MRS agar plates. Gram-staining and catalase test were performed to check on the purity of the isolates on both sub-cultures.

\section{Determination of colony-forming units} (CFU). The Miles and Misra method was applied to determine the colony-forming units. Lines were drawn on the bottom of agar plates to divide them into five equal parts [10]. On each part, $20 \mu \mathrm{L}$ of sample was pipetted on the surface of the agar. This was done in different plates with $10^{2}$ and $10^{4}$ dilutions for each LAB isolate, L. plantarum BS25 and S3 (KFR1) isolate. The plates were incubated at $37^{\circ} \mathrm{C}$ for $48 \mathrm{~h}$; the number of colonies in each section was counted. The $\mathrm{CFU} / \mathrm{mL}$ was computed from the section that yielded the highest number of distinct colonies.

Cholesterol-lowering property determination. Cholesterol solution $(3 \mathrm{mg} / \mathrm{mL})$ was prepared by diluting the cholesterol in $95 \%$ ethanol and Tween 80 with 3:1 ratio respectively. A $2 \mathrm{~mL}$ of filter-sterile cholesterol solution with $0.3 \%$ (w/v) ox bile was added to a $10 \mathrm{~mL}$ bacterial suspension. This solution was centrifuged at $6000 \mathrm{rpm}$ for $10 \mathrm{~min}$ and the absorbance of the supernatant was read at $500 \mathrm{~nm}$. The solution was vortexed at high speed before incubation at $37^{\circ} \mathrm{C}$ for $48 \mathrm{~h}$. After incubation, the solution was centrifuged and the absorbance of the supernatant was determined. Colorimetric cholesterol assay (CHOD-PAP) using Cholesterol Liquicolor Kit (Human Gesellschaft, Germany) was used to determined cholesterol concentration in the test solutions. Cholesterol concentration was computed based on manufacturer's protocol 
(amount of cholesterol $=200 \times($ Sample Absorbance/ Standard Absorbance).

Genomic DNA extraction, sequencing, and identification. Genomic DNA extraction of microorganisms was done using the Wizard $\AA$ Genomic DNA Purification Kit (Promega, U.S.A.). Five colonies were picked from MRS agar plates containing isolates with significant cholesterol-lowering activity and genomic DNA (gDNA) was extracted based on the protocol of the kit. After extraction, the gDNA was subjected to agarose gel electrophoresis to confirm successful extraction of gDNA. Extracted LAB gDNA was sent to Macrogen Korea for amplification and sequencing of the 16S rRNA gene. Sequence analysis using nucleotide BLAST was performed. The $754 \mathrm{bp}$ of the 16S rRNA gene of the identified LAB was deposited in GenBank.

\section{Results AND DISCUSSION}

Isolation of Lactobacillus. Samples and standard microorganisms are initially identified by morphological analysis. As seen in Fig. 1 microscopic analysis $(45 \times)$, both $L$. plantarum BS25 isolates and purified isolate from Philippine cocowater kefir were Grampositive, unciliated bacilli in colonies and chains and are catalase negative (result not shown). To further confirm the identity of both isolates, sample isolates were grown in MRS broth and agar, a selective media for lactic acid bacteria. Both isolate were milky white
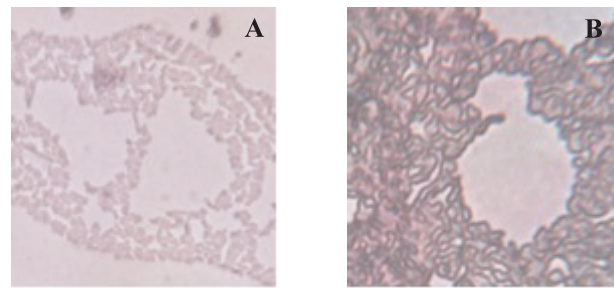

Figure 1. Microscopic analysis of Gram stained (A) L. plantarum BS25 (positive control) and (B) isolate S3 (L. plantarum KFR1) that exhibited cholesterol lowering property viewed under high power objectives. rounded colonies on the MRS agar confirming its identity as Lactobacillus species.

Cholesterol lowering activity. Isolates together with the positive control were subjected to Miles and Misra test [10] for cell viability. No observable colony growth was seen in negative control. Viable colonies of three isolates and positive control were counted as $3.75 \times 10^{10}$, $3.77 \times 10^{10}, 4.13 \times 10^{10}$, and $2.35 \times 10^{10} \mathrm{CFU} / \mathrm{mL}$ respectively (result not shown). The isolate with the most number of viable colonies in cocowater kefir was identified as L. plantarum KFR1.

The number of colony-forming units was determined in order to measure the extent of cholesterol-lowering ability of each isolate. For this reason, L. plantarum KFR1 including the standard were tested for cholesterol lowering activity using colorimetry. The cholesterol-lowering ability of each sample was based on the decrease of cholesterol concentration $(\mathrm{mg} / \mathrm{dL})$ after 24 and $48 \mathrm{~h}$ incubation.

The cholesterol concentration after 24-h incubation was $66.27 \mathrm{mg} / \mathrm{dL}$ and $29.00 \mathrm{mg} / \mathrm{dL}$ after $48 \mathrm{~h}$ incubation (Fig. 2). Lactobacillus plantarum KFR1 decreased cholesterol levels to 8.31 and $43.06 \mathrm{mg} / \mathrm{dL}$ at 24 and $48 \mathrm{~h}$ incubation (Fig. 3). One way analysis of variance and post hoc analysis using Dunett T3 test were done to determine cholesterol lowering at $24 \mathrm{~h}$ incubation among groups.

The statistical treatments on the cholesterol lowering activity of the controls and the isolate, L. plantarum KFR1 showed that cholesterol lowering after $24 \mathrm{~h}$ incubation between the groups and multiple comparison using Dunnett T3 was insignificant since the $p$-value generated was greater than the 0.05 significant level. And on the $48 \mathrm{~h}$ incubation, the cholesterol lowering of the coconut water, $L$. plantarum and positive control showed that 
there is a significant change $(p<0.05)$ with respect to the negative control. Statistical treatment done on the change of cholesterol level (Fig. 3) showed that within the group it was statistically significant $(p<0.05)$ but on the post-hoc analysis only the positive control showed significant lowering effect after 24hour incubation. After 48-hour incubation, coconut water, L. plantarum KFR1, and the positive control showed significant lowering on the cholesterol level tested in vitro $(p<0.05)$. Based on these results, it has been established that cholesterol level decreases as the incubation time increases.

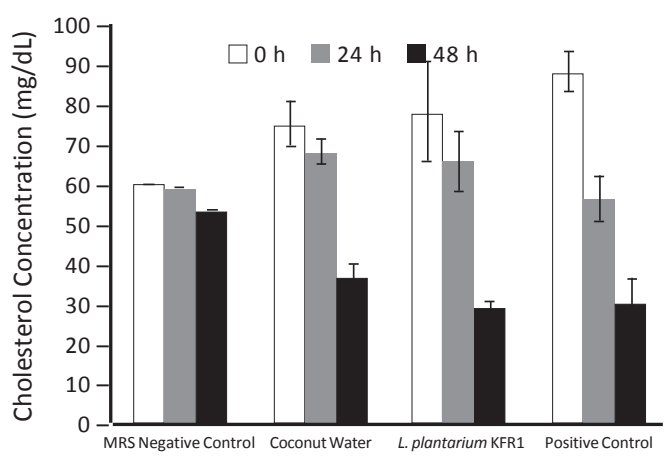

Figure 2. Cholesterol lowering activity in terms of cholesterol concentration of controls and $L$. plantarum KFR1 at three incubation time (0, 24, and $48 \mathrm{~h})$.

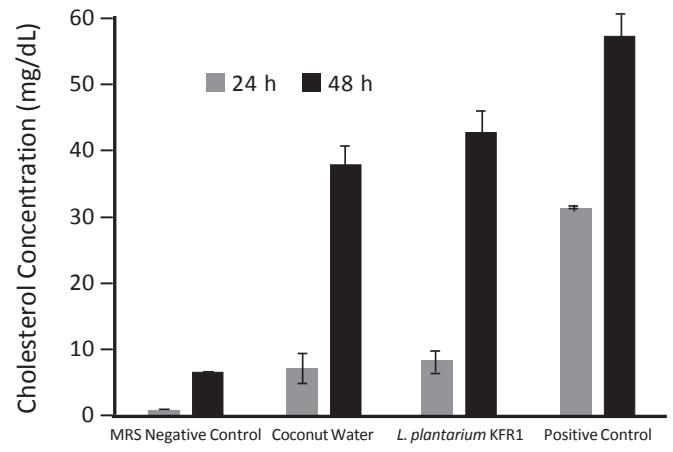

Figure 3. Change in the cholesterol lowering activity in terms of cholesterol concentration $(\mathrm{mg} / \mathrm{dL})$ of controls and L. plantarum KFR1 after 24 and $48 \mathrm{~h}$ incubation.
Coconut water has almost the same cholesterol lowering activity as that of the cholesterol lowering probiotic strain L. plantarum KFR1 (Fig. 2 and Fig. 3). This is because it is an abundant natural source of medium chain fatty acids (MCFA), with lauric acid (12-carbon fatty acid) being the most predominant. Also, polysaccharides present in coconut water can be fermented by other probiotics present in coconut water to produce short-chain fatty acids, vitamins, and other compounds [11]. which when present in significant amounts possess prebiotic properties that can also be attributed in enhancing the cholesterol lowering ability of the probiotic strain, L. plantarum KFR1 present in coconut water kefir to decrease cholesterol levels.

\section{Identity of $L A B$ with cholesterol-lowering activity. Extracted gDNA from the cocowater kefir isolates was visualized through agarose gel electrophoresis and compared with KAPA Universal Ladder (KAPA Biosystems) (Fig. 4). Gel viewed under UV showed that the position of its band aligns with that of the ladder at approximately $3000 \mathrm{bp}$. This size was within the range of that of previously isolated lactic acid bacteria gDNA (0.8-242 kbp) [12].}

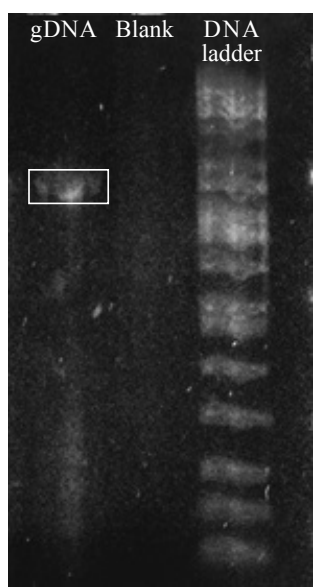

Figure 4. Agarose gel electropherogram of genomic DNA (gDNA) extracted from L. plantarum KFR1. 


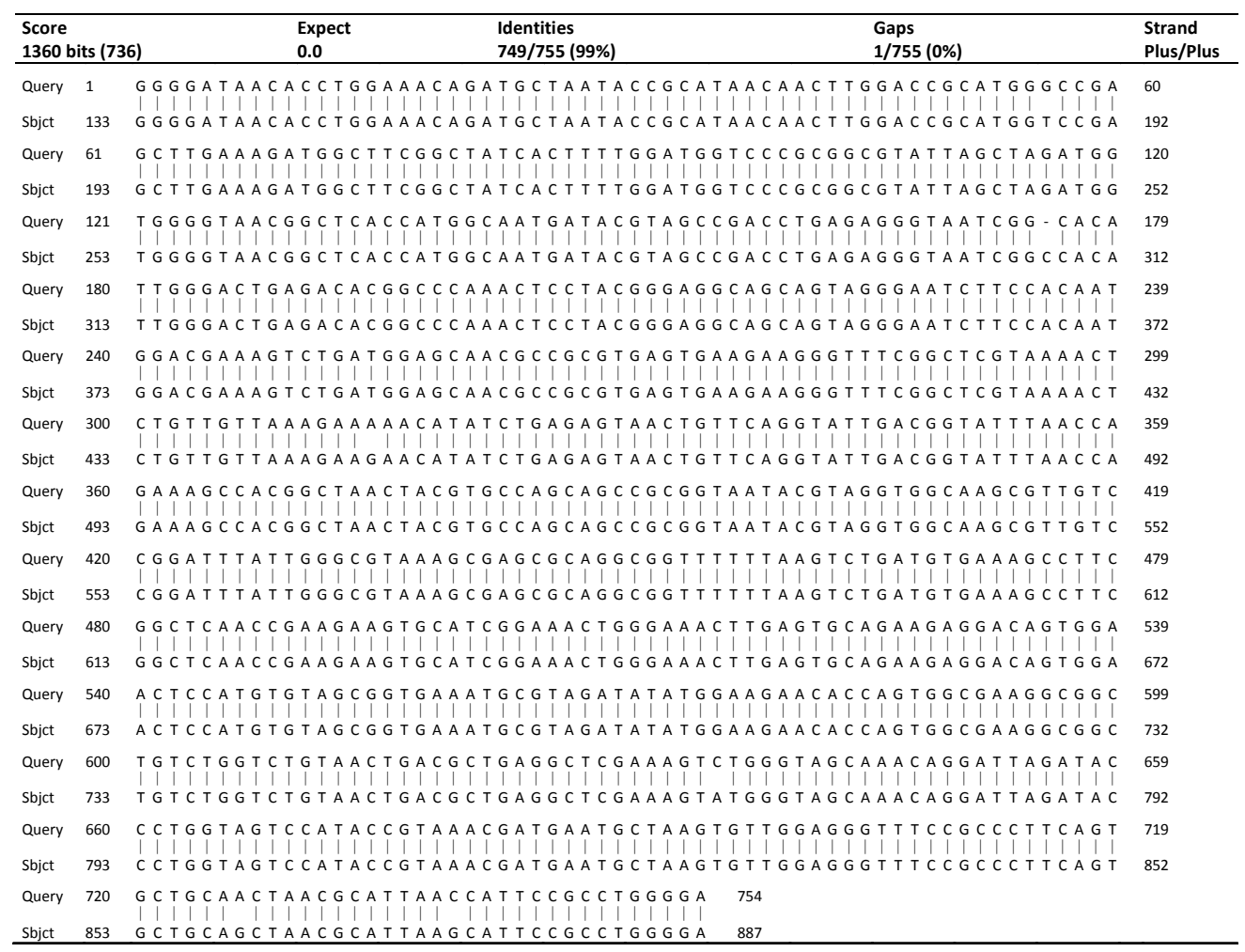

Figure 5. Sequence alignment of $\mathrm{S} 3$ isolate through BLAST, confirming its identity (99\%) as L. plantarum.

Sequence alignment of the cocowater kefir isolate (Fig. 5) confirmed that the isolate exhibit nearly the same cholesterol lowering activity as the standard, L. plantarum. It showed a $99 \%$ identity with the sequences in the GenBank. The 16S rRNA gene sequence of the identified bacteria was deposited at the GenBank with the definition of L. plantarum strain KFR1, 16S ribosomal RNA gene, partial sequence with an accession number of KT581997.

Other L. plantarum isolates have been shown to exhibit cholesterol-lowering such as $L$. plantarum I-UL4, isolated from food which showed significant decrease on the total cholesterol level of Sprague Dawley rats [13]. Some of the metabolites reported to be produced by the L. plantarum like orotic acid, uric acid, hydroxymethyl glutamic acid, and some mechanism that deconjugate bile salts tend to lower the plasma cholesterol level of an organism. Lactobacillus plantarum KLDS 1.0344 , which was isolated from homemade fermented cream in China exhibits high cholesterol removal and exhibits tolerance in $0.3 \%$ bile salts with using $8.56 \mathrm{CFU} / \mathrm{mL}$ for maximum activity $[13,14]$.

It was concluded that $L$. plantarum could be a potential additive to dietary supplements for lowering cholesterol levels in human [14]. There were three possible ways that the cholesterol can be lowered; first was the coprecipitation of cholesterol by means of bile salts deconjugation by bile salt hydrolase, second was by assimilation of cholesterol by the cells of microorganisms, and third was by 
degradation of cholesterol by the microorganisms for its nutritional requirement and or by other pathway that release it into the environment in degraded forms $[13,14]$.

Two strains of $L$. plantarum have been used in a previous study on the effect of the two strains on the lipid metabolism of high fat diet rats [15]. These two strains were L. plantarum LS/07 and L. plantarum Biocenol LP96. L. plantarum LS/ 07 showed reduction on the serum cholesterol level and the L. plantarum Biocenol LP96 decreased the triglycerides and VLDL levels. Both strains showed significantly lowered total bile acids in the serum but do not show any changes on the HDL and liver lipids [15].

\section{ACKNOWLEDGMENT}

Thanks to Andrea Vargas and Angelica Bernadette Crisostomo for their kindness in providing materials and equipment that were used in this study.

\section{REFERENCES}

[1] Anandharaj M \& Sivasankari B. Isolation of potential probiotic Lactobacillus oris HMI68 from mother's milk with cholesterol-reducing property. J. Biosci. Bioeng. 2014; 118(2):153-159.

[2] Gilliland SE, Nelson CR, \& Maxwell C. Assimilation of Cholesterol by Lactobacillus acidophilus. Appl. Environ. Microb. 1985; 49(2):377-381.

[3] du Toit M, Franz CM, Dicks LM, Schillinger U, Haberer P, Warlies B, Ahrens F, \& Holzapfel WH. Characterization and selection of probiotic lactobacilli for a preliminary minipig feeding trial and their effect on serum cholesterol levels, faeces $\mathrm{pH}$ and faeces moisture content. Int. J. Food Microbiol. 1998; 40(1-2): 93-104.

[4] Liong MT \& Shah NP. Acid and Bile Tolerance and Cholesterol Removal Ability of Lactobacilli Strains. J. Dairy Sci. 2005; 88:55-66.
[5] Lye HS, Rahmat-Ali GR, \& Liong MT. Mechanisms of cholesterol removal by lactobacilli under conditions that mimic the human gastrointestinal tract. Int. Dairy J. 2010; 20:169-175.

[6] Nguyen TDT, Kang JH, \& Lee MS. Characterization of Lactobacillus plantarum $\mathrm{PH} 04$, a potential probiotic bacterium with cholesterol-lowering effects. Int. J. Food Microbiol. 2007; 113:358-361.

[7] Huang Y, Wang X, Wang J, Wu F, Sui Y, Yang L, \& Wang Z. Lactobacillus plantarum strains as potential probiotic cultures with cholesterol-lowering activity. J. Dairy Sci. 2013; 96(5):2746-2753.

[8] Leite AMO, Miguel MAL, Peixoto RS, Rosado AS, Silva JT, \& Paschoalin VMF. Microbiological, technological and therapeutic properties of kefir: a natural probiotic beverage. Braz. J. Microbiol. 2013; 44(2):341-349.

[9] Prades A, Dornier M, Diop N, \& Pain JP. Coconut water uses, composition and properties: a review. Fruits. 2011; 67(2):87-107.

[10] Thoja TB, Izuka EH, Sikirat MO, Toyin AM, Omobowale AK, Oluwabunmi O, \& Oluwadun A. Enumeration of microorganism in dried cassava powder (Garri); a comparative study of four methods. New York Sci. J. 2012; 5(1):63-66.

[11] Thammarutwasik $P$, Hongpattarakere $T$, Chantachum S, Kijroongrojana K, Itharat A, Reanmongkol W, Tewtrakul S, \& Songklanakarin OB. Prebiotics - A Review. J. Sci. Technol. 2009; 31(4):401-408.

[12] Pfeiler EA \& Klaenhammer TR. The genomics of lactic acid bacteria. Trends Microbiol. 2007; 15(12):546-553.

[13] Foo HL, Loh TC, Lai PW, Lim YZ, Kufli CN, \& Rusul G. Effects of adding Lactobacillus plantarum I-UL4 metabolites in drinking water of rats. Pakistan J. Nutr. 2003; 2(5):283-288.

[14] Guo LD, Yang LJ, \& Huo GC. Cholesterol removal by Lactobacillus plantarum isolated from homemade fermented cream inner Mongolia of China. Czech. J. Food Sci. 2011; 29(3):219-225.

[15] Salaj R, Stofilova J, SoltesovaA, Hertelyova Z, Hijova E, Bertkova I, Strojny L, Kruzliak P, \& Bomba A. The effects of two Lactobacillus plantarum strains on rat lipid metabolism receiving a high fat diet. Sci. World J. 2013; 135142:1-7. 